\title{
Understanding kidney injury in COVID-19; a pressing priority
}

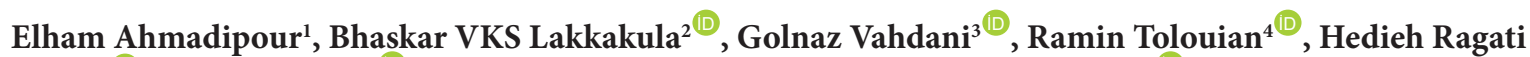

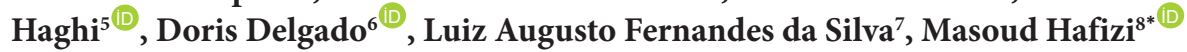

${ }^{1}$ Nickan Research Institute, Isfahan, Iran

${ }^{2}$ Department of Zoology, Guru Ghasidas Vishwavidyalaya, Bilaspur, India

${ }^{3}$ Division of Nephrology, University of Arizona, Tucson, AZ, USA

${ }^{4}$ Renal Section, Southern Arizona VA Health Care System, Tucson, AZ, USA

${ }^{5}$ Department of Radiology, Brigham and Women's Hospital, Boston, MA, USA.

${ }^{6}$ Independent Researcher and Consultant, 101 East Coso Street Suite 656 Ridgecrest, Ca, USA

${ }^{7}$ Departament of Nephrology, Life Center Hospital Belo Horizonte, Minas Gerais, Brazil

${ }^{8}$ Department of Infectious Diseases, Shahrekord University of Medical Sciences, Shahrekord, Iran

\section{A R T I C L E IN F O}

\section{Article Type:}

Mini Review

\section{Article History:}

Received: 16 October 2020

Accepted: 10 November 2020

ePublished: 26 November 2020

\section{Keywords:}

COVID-19, Kidney injury,

Renal transplant, Hemodialysis,

Angiotensin-converting enzyme 2,

Cytokine storm, SARS-CoV-2, Acute

kidney injury

\begin{abstract}
A B S T R A C T
The 2019 novel coronavirus disease (COVID-19) is a newly defined infectious and highly contagious acute disease caused by the severe acute respiratory syndrome coronavirus 2 ( (SARSCoV-2). COVID-19 is mainly characterized by an acute respiratory disease however it can also affect multiple other organ systems such as the kidney, gastrointestinal tract, heart, vascular system, and the central nervous system. Kidney involvement is frequent in patients with COVID-19 and this review aims to explore the available data on kidney and COVID-19. In conclusion, COVID-19 infection can affect renal function and may cause acute kidney injury (AKI), due to several mechanisms that need to be fully elucidated. As only supportive management strategies are available for treating AKI in COVID-19, it is necessary to identify and preserve renal function during SARS-CoV-2 infection.
\end{abstract}

Implication for health policy/practice/research/medical education:

Numerous studies have shown that, acute kidney injury was independently associated with higher mortality in COVID-19.

Please cite this paper as: Ahmadipour E, Lakkakula BVKS, Vahdani G, Tolouian R, Ragati Haghi H, Delgado D, da Silva LAF,

Hafizi M. Understanding kidney injury in COVID-19; a pressing priority. J Nephropharmacol. 2021;10(2):e19. DOI: 10.34172/ npj.2021.19.

\section{Introduction}

Novel coronavirus disease (COVID-19) is a newly discovered serious infectious and highly contagious acute disease caused by SARS-CoV-2 (severe acute respiratory syndrome coronavirus 2). It first appeared in Wuhan, China, in early December 2019, and rapidly evolved into a global pandemic recognized by the World Health Organization (WHO). COVID-19 can be transmitted directly from human to human via contact or respiratory droplets. COVID-19 is characterized by acute respiratory disease, with most patients $(80 \%)$ presenting mild and self-limited flu-like symptoms, however $20 \%$ may have a severe presentation with acute respiratory distress syndrome (ARDS) (1). The first phase of the disease comprises an incubation period for individuals infected with COVID-19 that may vary from 1 to 14 days (most commonly, 3-10 days). The second phase of the disease, which is related closely to the risk of mortality, usually begins between days 7 to 10 from the onset of symptoms that is associated with growing requirements of oxygen supply and respiratory support. This condition is likely to be secondary to hyper inflammatory and cytokine release syndromes (2).

Although, COVID-19 is characterized by interstitial and alveolar pneumonia, it can also affect multiple other organ systems such as kidney, gastrointestinal tract, 
heart, vascular system, and the central nervous system. The clinical spectrum of COVID-19 infection ranges from asymptomatic infection, mild upper respiratory tract disease to severe viral pneumonia with respiratory failure and death (3). The risk factors for severe disease and mortality still have not been fully defined (4). Studies thus far demonstrated that underlying disease and comorbidities such as diabetes mellitus, cardiovascular disease, congestive heart failure, renal failure, cancer, hypertension, high body mass index and obstructive pulmonary disease could affect survival of the patients infected by COVID-19. Additionally, these factors can also affect the duration of hospitalization and recovery process from infection (5). The co-morbidities that increase the risk of mortality in patients with SARS-CoV-2 are common in patients with chronic kidney disease (CKD) and endstage renal disease (ESRD) (6). Immunosuppressive agents are crucial for successful kidney transplantation; however they may enhance the risk of complications in COVID-19 infection. Further, recent reports suggest that kidney transplant patients showed a more severe disease course and requiring intensive care admission (7).

Coronavirus is developing some unique adaptations through mutations and recombination that provide the virus with the special ability to bind to ACE2 (angiotensinconverting enzyme 2). ACE2 is present in the epithelium of lung, small intestine and urogenital cells. In the kidneys ACE2 is expressed mainly in proximal tubules, afferent arterioles, collecting ducts and the thick ascending limb of Henle (1). Therefore, in addition to fever and respiratory complications, it may cause acute kidney injury (AKI) that is presented with an increase in serum creatinine level and a decrease in urine output. It was recently demonstrated that SARS-CoV2 may induce tubular necrosis by infecting the kidney tubules directly (1). An epidemiological study suggested that $36.6 \%$ patients with COVID-19 had different degrees of AKI (8). Several lines of evidence demonstrate that the host cells facilitate virus entry through ACE2 receptors in epithelial cells. In addition, transmembrane serine protease 2 (TMPRSS2) prime the coronavirus spike protein, which is a determining factor of SARS-CoV-2 entry in host cells. Similarly, the ACE2 and TMPRSS2 genes were significantly co-expressed in podocytes and proximal tubule cells, while these two types of cells have the most significant effect on filtration, reabsorption and excretion activity. Meanwhile, podocytes are also sensitive to the direct damage by viral infections like COVID-19, which may lead to proteinuria $(9,10)$.

It has been reported that $8.3 \%-29 \%$ of intensive care unit (ICU) patients develop AKI during COVID-19 (10) with higher rates reported in countries outside of China (4-8).

Patients with COVID-19 may present to medical care with AKI or develop it during their hospitalization, with an average of 5 to 9 days after admission. Those requiring admission to the ICU have a higher rate of AKI than those hospitalized on the wards. AKI is also a well recognized factor of poor prognosis $(2,11)$.

The cause of AKI in COVID-19 is likely multi-factorial with predisposing factors, such as sepsis, hypovolemia, hypotension, hypoxemia, mechanical ventilation, high PEEP, nephrotoxins all being important contributors. Direct viral cytopathic effect, microvascular thrombosis and increased pro-inflammatory cytokines release, proteinuria in $63 \%$ of patients with a median of 20 days after onset of clinical symptoms was detected (12). The possible mechanism of renal injury may be due to initiation of kidney cell apoptosis by overexpression of FGF2 (fibroblast growth factor 2) and SMAD7 (SMAD family member 7) during SARS-CoV-2 infection (13). The study by Ling et al detected the positive viral RNA (ribonucleic acid) in urine samples of individuals with SARS-CoV-2 (14). Therefore, renal injury can be related to cytopathic effect of COVID-19 that is mediated by cytokine storm syndrome and possible direct viral invasion to kidney cells. The systemic inflammatory response as a cause of cytokine storm syndrome mediates a drastic increase of interleukin-2, interleukin-7, interleukin-10, granulocyte colony stimulating factor, interferon gamma induced protein 10, monocyte chemoattractant protein-1, macrophage inflammatory protein 1alpha and tumor necrosis factor alpha. In this scenario, hyperactive immune response is more likely to attack human cells rather than the virus, which partially explains the course of renal injury during SARS-CoV-2 infection (12-15).

Clinical findings of 138 hospitalized individuals with SARS-CoV-2 in China revealed that the co-morbidities are more prevalent among patients who were admitted in ICU (72.2\%) as compared to non-ICU patients (37.3\%) (16). Mortality in patients with renal transplants or on chronic hemodialysis with COVID-19 infection is substantial. In a group of 20 individuals with renal transplants and SARSCoV-2 almost 25\% died and 15\% were discharged after 13 days. In the dialysis population, $23 \%$ died and $19 \%$ were discharged between 7-17 days. Elderly patients on chronic hemodialysis with multiple co-morbidities had higher mortality in ICU setting (15-17).

Immune responses induced by COVID-19 infection have two phases, incubation phase and severe phase. The first phase is closely related to the genetic background of host immune pathways. If the immune system is not potent enough to eradicate the virus attack, this infection leads to an extensive damage of tissues, which then expresses ACE2 receptors in lung, intestine and kidney tissues (18). Incubation stage causes immune inflammatory response through macrophages and granulocytes. The second phase, the severe phase related to systemic cytokine storm such as, lymphocytopenia, in addition to lung and kidney tissues damage (19). In a nutshell, the incubation phase is an immune defense-based or protective phase and the severe phase is an inflammation-driven or damaging phase. Hence, treatment during the first phase should be adapted towards strengthening the patient's immune system, while 
immunosuppression is necessary during the second phase of the disease (15). Additionally, a hypercoagulable state is frequently observed in COVID-19 patients with severe disease (2).

Histological examination of renal tissues from postmortem samples found acute renal tubular injury in six confirmed COVID-19 cases (20). The inflammatory cytokines that are produced during lung injury can cause damage to the kidneys. Further, kidney damage can hasten the inflammation response, which was established in the lung. Alternatively, the damage and destruction of renal tubular epithelial cells and podocytes can lead to damage of the pulmonary system and other organs by release of numerous inflammatory cytokines, which is defined as cytokine release syndrome (20). However, another study showed, no noticeable renal dysfunction was observed in patients with COVID-19 pneumonia (12). Likewise, analysis of clinical characteristics of 138 hospitalized COVID-19 patients found that the levels of creatinine and blood urea nitrogen were in normal range (16). Similarly, analysis of laboratory data from 1099 confirmed COVID-19 patients revealed that the AKI was uncommon in COVID-19 patients (21). Recent autopsy data demonstrated that tubular epithelial cells necrosis and degeneration, with interstitial hyperemia, microthrombi or focal fibrosis were the main pathological features, while glomerular lesions are less common with case reports of collapsing glomerulopathy $(1,22)$.

Individuals on dialysis with SARS-CoV-2 had less lymphopenia, lower serum levels of inflammatory cytokines, and milder clinical disease than other patients with COVID-19 infection. However, COVID-19 infection is a serious threat for patients undergoing dialysis as well as staff who are working in hemodialysis centers. Data from a solitary hemodialysis center in Renmin hospital, Wuhan University showed that 37 out of 230 patients on hemodialysis and 4 of 33 staff members were infected with COVID-19 between January 14 and February 17, 2020 (23).

Like in other viral infections, some cases of glomerulonephritis (GN) were also reported in patients with SARS-CoV-2 infection. Although the precise mechanisms are not known, there is no doubt that inflammation-induced podocyte injury due to cytokine release syndrome play a crucial role in GN (24). Further, histopathological examinations demonstrated viral particles in the cytoplasm of podocytes of patients with GN (25). It is also interesting to note that all GN COVID-19 cases are related to African origin and carrying APOL1 genotypes (26-28).

\section{Treatment of COVID-19 patients with AKI}

The existing treatment of COVID-19 patients with AKI contains general, supportive management and renal replacement therapy. Currently there are no specific antiviral therapies or monoclonal antibodies against
COVID-19. Continuous renal replacement therapy may be useful in critically ill patients suffering with AKI or patients having hyperactive immune response (29). Further, extracorporeal renal replacement therapy may be used to achieve immune homeostasis. Furthermore, kidney injury also affects the metabolism and excretion of drugs prescribed in COVID-19 infection. In this regard, most of the metabolites derived from oseltamivir, ribavirin and some of the metabolites of lopinavir/ritonavir are detected in the urine of these patients. Therefore, inappropriate doses of these medications may lead to kidney damage and nephrotoxicity (28-31).

\section{Conclusion}

In conclusion, COVID-19 infection can affect renal function and may cause kidney injury due to several mechanisms that are not yet fully understood. The most identified mechanism is cytokine-release syndrome. However, hypoxemia during infection may lead to multi-organ damage including kidney injury. Noticeably, COVID-19 patients with underlying kidney insufficiency have higher risk of mortality. AKI was independently associated with higher mortality, to date; only supportive management strategies are available for AKI in COVID-19.

\section{Authors' contribution}

$\mathrm{EH}$ and $\mathrm{MH}$ prepared the primary draft. BVKSL performed the first edit. GV, RT, HRH, DD and LAFdS performed the final edit and provided critical feedback. All authors read and approved the final manuscript.

\section{Conflicts of interest}

The authors declared no potential conflicts of interest with respect to the research, authorship, and/or publication of this article.

\section{Ethical considerations}

Ethical issues (including plagiarism, data fabrication, double publication) have been completely observed by the authors.

\section{Funding/Support}

None.

\section{References}

1. Qian J, Wang B, Liu B. Acute kidney injury in the 2019 novel coronavirus disease. Kidney Dis. 2020;6:318-23. doi: 10.1159/000509086.

2. Zhou F, Yu T, Du R, Fan G, Liu Y, Liu Z, et al. Clinical course and risk factors for mortality of adult in-patients with COVID-19 in Wuhan, China: a retrospective cohort study. Lancet. 2020;395:1054-62. doi: 10.1016/S01406736(20)30566-3.

3. Yang J, Zheng Y, Gou X, Pu K, Chen Z, Guo Q, et al. Prevalence of comorbidities and its effects in patients infected with SARS-CoV-2: a systematic review and meta-analysis. Int J Infect Dis. 2020;94:91-5. doi: 10.1016/j.ijid.2020.03.017. 
4. Chen J. Pathogenicity and transmissibility of 2019-nCoV-A quick overview and comparison with other emerging viruses. Microbes Infect. 2020;22:69-71. doi: 10.1016/j. micinf.2020.01.004.

5. Li X, Xu S, Yu M, Wang K, Tao Y, Zhou Y, et al. Risk factors for severity and mortality in adult COVID-19 inpatients in Wuhan. J Allergy Clin Immunol. 2020;146:110-118. doi: 10.1016/j.jaci.2020.04.006.

6. Cheng Y, Luo R, Wang K, Zhang M, Wang Z, Dong L, et al. Kidney disease is associated with in-hospital death of patients with COVID-19. Kidney Int. 2020;97:829-38. doi: 10.1016/j.kint.2020.03.005.

7. Banerjee D, Popoola J, Shah S, Ster IC, Quan V, Phanish M. COVID-19 infection in kidney transplant recipients. Kidney Int. 2020;97:1076-82. doi: 10.1016/j.kint.2020.03.018.

8. Hirsch JS, Ng JH, Ross DW, Sharma P, Shah HH, Barnett RL, et al. Acute kidney injury in patients hospitalized patients with;COVID-19. Kidney Int. 2020;98:209-18. doi: 10.1016/j. kint.2020.05.006.

9. Su H, Yang M, Wan C, Yi L-X, Tang F, Zhu H-Y, et al. Renal histopathological analysis of 26 postmortem findings of patients with COVID-19 in China. Kidney Int. 2020;98:21927. doi: 10.1016/j.kint.2020.04.003.

10. Shang Y, Pan C, Yang X, Zhong M, Shang X, Wu Z, et al. Management of critically ill patients with COVID-19 in ICU: statement from front-line intensive care experts in Wuhan, China. Ann Intensive Care. 2020;10:73-. doi: 10.1186/ s13613-020-00689-1.

11. Asgharpour M, Zare E, Mubarak M, Alirezaei A. COVID-19 and kidney disease: update on epidemiology, clinical manifestations, pathophysiology and management. J Coll Physicians Surg Pak. 2020;30:19-25. doi: 10.29271/ jcpsp.2020.Supp1.S19.

12. Wang L, Li X, Chen H, Yan S, Li D, Li Y, et al. Coronavirus Disease 19 infection does not result in acute kidney injury: an analysis of 116 hospitalized patients from Wuhan, China. Am J Nephrol. 2020;51(5):343-348. doi: 10.1159/000507471.

13. Pan XW, Xu D, Zhang H, Zhou W, Wang LH, Cui XG. Identification of a potential mechanism of acute kidney injury during the COVID-19 outbreak: a study based on single-cell transcriptome analysis. Intensive Care Med. 2020;46:1114-6. doi: 10.1007/s00134-020-06026-1.

14. Ling Y, Xu SB, Lin YX, Tian D, Zhu ZQ, Dai FH, et al. Persistence and clearance of viral RNA in 2019 novel coronavirus disease rehabilitation patients. Chin Med J (Engl). 2020;133:1039-43. doi: 10.1097/CM9.0000000000000774.

15. Shi Y, Wang Y, Shao C, Huang J, Gan J, Huang X, et al. COVID-19 infection: the perspectives on immune responses. Cell Death Differ. 2020;27:1451-4. doi: 10.1038/s41418-0200530-3.

16. Wang D, Hu B, Hu C, Zhu F, Liu X, Zhang J, et al. Clinical Characteristics of 138 Hospitalized Patients With 2019 Novel Coronavirus-Infected Pneumonia in Wuhan, China. JAMA. 2020;323:1061-9. doi: 10.1001/jama.2020.1585.

17. Alberici F, Delbarba E, Manenti C, Econimo L, Valerio F, Pola A, et al. Management of patients on dialysis and with kidney transplantation during the SARS-CoV-2 (COVID-19) pandemic in Brescia, Italy. Kidney Int Rep. 2020;5:580-5. doi: 10.1016/j.ekir.2020.04.001.

18. Ryan PM, Caplice N. COVID-19 and relative angiotensinconverting enzyme 2 deficiency: role in disease severity and therapeutic response. Open Heart. 2020;7:e001302. doi: 10.1136/openhrt-2020-001302.

19. Sun X, Wang T, Cai D, Hu Z, Chen Ja, Liao H, et al. Cytokine storm intervention in the early stages of COVID-19 pneumonia. Cytokine Growth Factor Rev. 2020;53:38-42. doi: 10.1016/j.cytogfr.2020.04.002.

20. Diao B, Wang C, Wang R, Feng Z, Tan Y, Wang H, et al. Human kidney is a target for novel severe acute respiratory syndrome coronavirus 2 (SARS-CoV-2) Infection. medRxiv. 2020:2020.03.04.20031120. doi: 10.1101/2020.03.04.20031120.

21. Guan W-j, Ni Z-y, Hu Y, Liang W-h, Ou C-q, He J-x, et al. Clinical characteristics of 2019 novel coronavirus infection in China. medRxiv. 2020:2020.02.06.20020974. doi: 10.1101/2020.02.06.20020974.

22. National Health Comission of the Peoples's Republic of China. Guidelines for the diagnosis and treatment of 2019 novel coronavirus disease (version 7). National Health Commission of the Peoples's Republic of China; 2020.

23. Ma Y, Diao B, Lv X, Zhu J, Liang W, Liu L, et al. COVID-19 in hemodialysis (HD) patients: Report from one HD center in Wuhan, China. medRxiv. 2020:2020.02.24.20027201. doi: 10.1101/2020.02.24.20027201.

24. Nasr SH, Kopp JB. COVID-19; Associated Collapsing Glomerulopathy: An Emerging Entity. Kidney Int Rep. 2020;5:759-61. doi: 10.1016/j.ekir.2020.04.030.

25. Golmai P, Larsen CP, DeVita MV, Wahl SJ, Weins A, Rennke HG, Bijol V, Rosenstock JL. Histopathologic and ultrastructural findings in postmortem kidney biopsy material in 12 patients with AKI and COVID-19. J Am Soc Nephrol. 2020;31:1944-7. doi: 10.1681/ASN.2020050683.

26. Larsen CP, Bourne TD, Wilson JD, Saqqa O, Sharshir MdA. Collapsing glomerulopathy in a patient with coronavirus disease 2019 (COVID-19). Kidney Int Rep. 2020;5:935-9. doi: 10.1016/j.ekir.2020.04.002.

27. Kissling S, Rotman S, Gerber C, Halfon M, Lamoth F, Comte $\mathrm{D}$, et al. Collapsing glomerulopathy in a COVID-19 patient. Kidney Int. 2020. doi: 10.1016/j.kint.2020.04.006.

28. Peleg Y, Kudose S, D’Agati V, Siddall E, Ahmad S, Kisselev S, et al. Acute kidney injury due to collapsing glomerulopathy following COVID-19 infection. Kidney Int Rep. 2020;5:9405. doi: 10.1016/j.ekir.2020.04.017.

29. Adapa S, Aeddula NR, Konala VM, Chenna A, Naramala S, Madhira BR, et al. COVID-19 and renal failure: challenges in the delivery of renal replacement therapy. J Clin Med Res. 2020;12:276-85. doi: 10.14740/jocmr4160.

30. Makris K, Spanou L. Acute kidney injury: definition, pathophysiology and clinical phenotypes. Clin Biochem Rev. 2016;37:85-98.

31. Li Z, Wu M, Yao J, Guo J, Liao X, Song $\mathrm{S}$, et al. Caution on Kidney Dysfunctions of COVID-19 Patients. medRxiv. 2020:2020.02.08.20021212. doi: $10.1101 / 2020.02 .08 .20021212$.

Copyright (c) 2021 The Author(s); Published by Published by Society of Diabetic Nephropathy Prevention. This is an open-access article distributed under the terms of the Creative Commons Attribution License (http://creativecommons.org/licenses/by/4.0), which permits unrestricted use, distribution, and reproduction in any medium, provided the original work is properly cited. 